\title{
Magnetic states of an individual Ni nanotube probed by anisotropic magnetoresistance
}

\author{
Daniel Rüffer, ${ }^{a}$ Rupert Huber, ${ }^{b}$ Paul Berberich, ${ }^{b}$ Stephan Albert, ${ }^{b}$ Eleonora Russo-Averchi, ${ }^{a}$ Martin Heiss, ${ }^{a}$ \\ Jordi Arbiol, $^{c}$ Anna Fontcuberta i Morral ${ }^{\star * a}$ and Dirk Grundler ${ }^{b d}$
}

\author{
Received 8th March 2012, Accepted 31st May 2012 \\ DOI: $10.1039 / \mathrm{c} 2 \mathrm{nr} 31086 \mathrm{~d}$
}

\begin{abstract}
Defined magnetization states in magnetic nanotubes could be the basic building blocks for future memory elements. To date, it has been extremely challenging to measure the magnetic states at the single-nanotube level. We investigate the magnetization states of an individual Ni nanotube by measuring the anisotropic magnetoresistance effect at cryogenic temperature. Depending on the magnitude and direction of the magnetic field, we program the nanotube to be in a vortex- or onion-like state near remanence.
\end{abstract}

\section{Introduction}

Ferromagnetic top-down and bottom-up nanostructures constitute the basic building blocks for future high-density memory elements. They are an alternative to the current planar technology which is expected to face fundamental physical limitations in the next few years. ${ }^{1}$ Three-dimensional architectures based on ferromagnetic nanowires have been proposed to overcome the limits. ${ }^{2}$ Especially interesting is the application of nanoscale ferromagnetic materials in magnonic devices. ${ }^{3,4}$ There, the control and manipulation of spin waves at the nanoscale are expected to offer novel perspectives for data transmission ${ }^{5}$ and data processing. ${ }^{6}$ At the same time, magnetic nanoparticles and disks are powerful materials for biological applications, drug delivery, targeted magnetic resonance imaging and magnetothermal treatment of tissues. ${ }^{7-10}$ Low-dimensional ferromagnetic nanostructures are particularly interesting due to unique magnetic configurations. ${ }^{11-16}$ In contrast to nanowires or dots, hollow nanotubes possess three independent geometrical parameters for the control of the magnetic properties via shape anisotropy, i.e., the length $L$, the inner radius $r_{\mathrm{i}}$ and the outer radius $r_{\mathrm{o}}$. It has been predicted that the magnetization reversal via vortex wall formation and propagation might be more controlled in nanotubes compared to solid nanowires since in

${ }^{a}$ Laboratoire des Matériaux Semiconducteurs, Institut des Matériaux, Ecole Polytechnique Fédérale de Lausanne, 1015 Lausanne, Switzerland. E-mail: anna.fontcuberta-morral@epfl.ch; Fax: +41 21693 4755; Tel: +41216937394

${ }^{b}$ Lehrstuhl für Physik funktionaler Schichtsysteme, Physik Department, Technische Universität München, James-Franck-Str. 1, 85747 Garching bei München, Germany

'Institució Catalana de Recerca i Estudis Avançats (ICREA), Institut de Ciència de Materials de Barcelona (ICMAB-CSIC), Campus de la UAB, 08193 Bellaterra, Spain

${ }^{d}$ STI, Faculté Sciences et Technique de l'Ingénieur, Ecole Polytechnique Fédérale de Lausanne, 1015 Lausanne, Switzerland nanotubes the Bloch point structure is avoided. ${ }^{17}$ Numerous theoretical predictions exist concerning remanent states of an individual ferromagnetic nanotube. ${ }^{18-23}$ So far, however, only large ensembles of nanotubes have been studied experimentally which were fabricated from a ferromagnetic metal. ${ }^{24-31}$ Not only the nanotubes exhibited different diameters, but also a different orientation with respect to the magnetic field $\boldsymbol{H}$. All this led to magnetic hysteresis curves that were difficult to interpret. Recently an individual nanotube of GaMnAs was studied. ${ }^{32}$ There, magnetocrystalline anisotropy of the ferromagnetic semiconductor dominated over the shape anisotropy. This does not allow one to address the peculiar magnetic states of a nanotube. In this paper we report an experimental study performed on individual nanotubes that have been fabricated from a metallic ferromagnet. The nanotubes consist of a $40 \mathrm{~nm}$ thick $\mathrm{Ni}$ layer deposited by Atomic Layer Deposition (ALD) on GaAs nanowires as nano-templates. In particular the nickel film is polycrystalline and does not exhibit magnetocrystalline anisotropy. The use of ALD on self-assembled nanowires enables one to reach unprecedented aspect ratios and thereby tailor shape anisotropy in magnetic systems. In this study, the nanotubes have a diameter of $150 \mathrm{~nm}$ and a length of $20 \mu \mathrm{m}$. The nanotubes are straight and mechanically robust as they are supported by the insulating GaAs nanowire core. This is an ideal configuration for the integration of electrical contacts and the measurement of the anisotropic magnetoresistance (AMR) effect. The AMR effect is a powerful tool to study the magnetization states of individual nanomagnets as demonstrated on planar nanostripes ${ }^{33}$ and solid nanowires. ${ }^{34}$ Following the theory of AMR in thin metallic films ${ }^{35}$ we discuss a classification of relevant nanotube magnetization states in terms of the relative AMR effect for the first time. This allows us to analyze the magnetization reversal under the two orthogonal orientations of $\boldsymbol{H}$ parallel and perpendicular to the nanotube axis. For both orientations, segments of the nanowires are found to align their remanent magnetization in the 
azimuthal direction. The magnetoresistance traces for perpendicular field orientation suggest the transition from an onion-like state to a vortex configuration in opposing field.

\section{Sample fabrication and thin-film properties}

The magnetic nanotubes were fabricated using a two step process. In the first step, GaAs nanowires were grown on a $2^{\prime \prime}$ Si(111) substrate in the self-catalyzed growth mode. The growth was performed in a DCA P600 molecular beam epitaxy (MBE) system. Si wafers were used without removing the native oxide before starting the growth. After the axial growth, the mode was switched to planar growth in order to deposit an epitaxial shell of GaAs and thus increase the diameter in a controlled manner. Further details on the nanowire growth can be found elsewhere. ${ }^{36,37}$ Finally, the nanowires were coated with roughly $2 \mathrm{~nm}$ of aluminum to protect the wires from decomposition at the elevated temperatures needed for the subsequent atomic layer deposition (ALD) of nickel oxide in the second step. For this, the samples were transferred to an ALD vacuum chamber PicoSun Sunale $\mathrm{P}$, where first a $25 \mathrm{~nm}$ thick layer of $\mathrm{Al}_{2} \mathrm{O}_{3}$ was deposited ${ }^{38}$ using trimethylaluminium and water. This layer of $\mathrm{Al}_{2} \mathrm{O}_{3}$ was conformally grown around the nanowires to isolate the core and prevent arsenic from diffusing into the nickel nanotube. Second, the nanowires were exposed to successive pulses of nickelocene NiCp 2 , ozone $\left(\mathrm{O}_{3}\right)$ and hydrogen $\left(\mathrm{H}_{2}\right)$. The substrate was held at $300^{\circ} .{ }^{39}$ We used 800 cycles to form a $40 \mathrm{~nm}$ thick nickel oxide layer which was partially reduced by the hydrogen pulses. To further improve the reduction of nickel oxide to metallic nickel the sample was held at $350{ }^{\circ} \mathrm{C}$ for four hours in a hydrogen atmosphere.

The ferromagnetic behavior was studied by ferromagnetic resonance (FMR) measurements at room temperature performed on planar reference films grown by the same ALD process. The FMR data showed a pronounced resonance line varying characteristically with the applied magnetic field. ${ }^{39}$ The FMR data did not depend on the orientation of the in-plane magnetic field. This behavior suggests vanishing magnetocrystalline anisotropy of the ALD-grown Ni. This is attributed to the polycrystalline nature (see Section 3 ) and is in contrast to GaMnAs used in ref. 32.

AMR measurements on ALD-grown planar Ni films of $10 \mathrm{~nm}$ thickness showed a relative MR effect of about $0.7 \%$ at $4.2 \mathrm{~K}$.

$$
\operatorname{MR}=\left(\rho_{\|}-\rho_{\perp}\right) / \rho_{\perp}
$$

Here $\rho_{\|}\left(\rho_{\perp}\right)$ is the specific resistivity for the device being saturated in the direction of (perpendicular to) the current $I$. In this case, $\rho_{\perp}$ was taken with $\boldsymbol{H}$ being perpendicular to the plane. The shape anisotropy field of plain Ni films amounted to about $0.4 \mathrm{~T}$ in perpendicular field. ${ }^{40}$

\section{Nanotube characterization}

\subsection{Structure and composition}

We proceed now with the presentation of the structure and composition of the magnetic nanotubes which were grown as a Ni shell on a core consisting of a $150 \mathrm{~nm}$ diameter semi-insulating GaAs nanowire. A Scanning Electron Microscopy (SEM) image of a core-shell device is shown in Fig. 1(a). The morphology and conformal nature of the Ni layer is extracted from Fig. 1(b), where a Scanning Transmission Electron Microscopy (STEM) High Angle Annular Dark Field (HAADF) image is shown. We find nickel of an average thickness of about $40 \mathrm{~nm}$ along up to $20 \mu \mathrm{m}$ long GaAs nanowires and around the Ga nanodroplets used to grow the semiconductor cores. The nickel shell is polycrystalline and exhibits some remaining nanotroughs. By SEM investigations we observed that the roughness depended on the diameter of the GaAs nanowires forming the templates for the ALD growth process. The roughness is due to the Ni deposition, as the GaAs nanowires themselves exhibit planar facets with a roughness on the atomic scale. ${ }^{41} \mathrm{We}$ attribute the formation of nanotroughs to surface tension effects in the hydrogen-based reduction process after the ALD growth. As a consequence, the roughnesses of nanotubes and planar reference films are also found to differ. Randomly oriented grain boundaries are expected to exist in the Ni shell. Profiles obtained in the HAADF STEM images provide the expected hexagonal cross-section of the GaAs core, ${ }^{42}$ and a quasi-spherical shell. Electron Energy
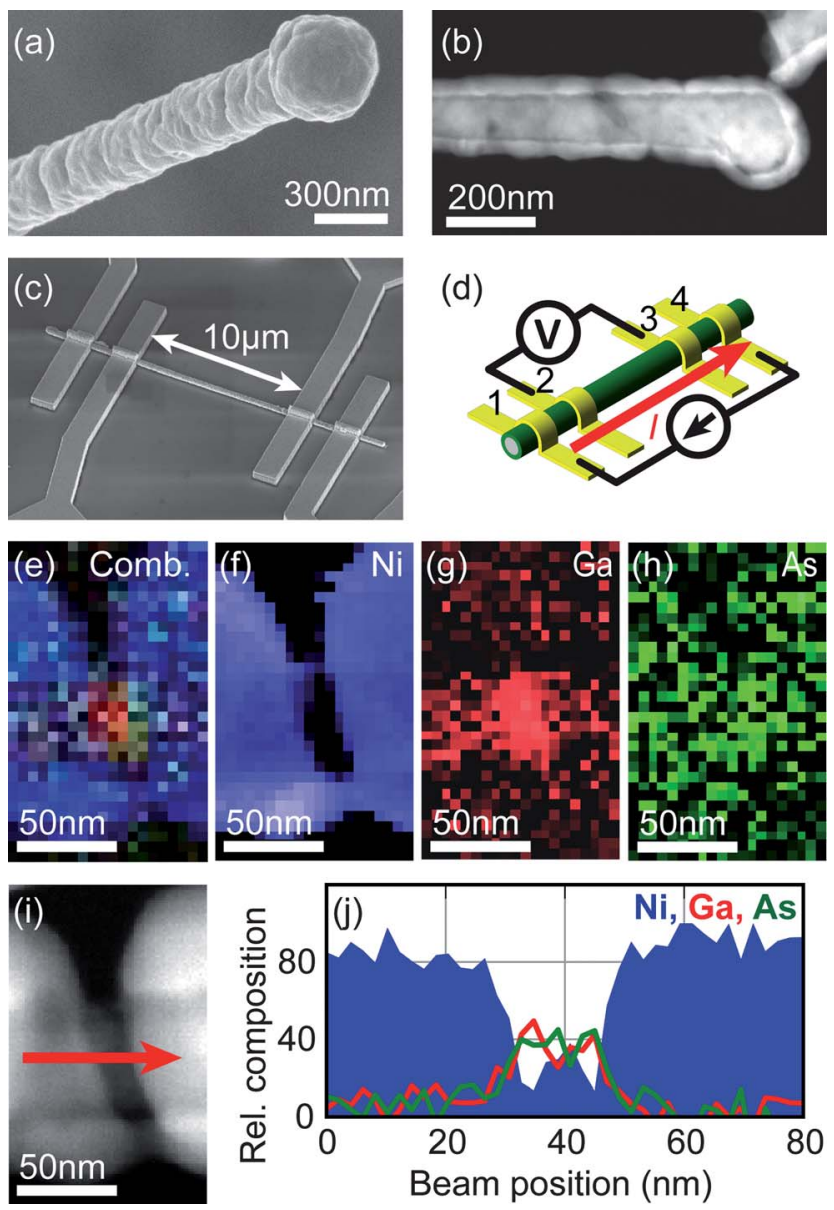

Fig. 1 (a) SEM and (b) HAADF STEM images of a magnetic nanotube. We find the nickel to conformally overgrow both the GaAs nanowire and the nanodroplet used for epitaxial growth of the core. (c and d) $\mathrm{A} \mathrm{Ni}$ nanotube of a length of about $20 \mu \mathrm{m}$ contacted by Au leads in a fourpoint configuration. (e-h) EELS images for the area around a nanotrough (i). The EELS profile (j) was obtained along the red arrow direction. 
Loss Spectroscopy (EELS) images shown in Fig. 1(e-h) confirm the presence of $\mathrm{Ni}$ in the extremal shell of the nanotube on the GaAs core. Due to the high electron scattering on the Ni shell, it was very difficult to obtain appropriate EELS maps. As seen above, the Ni shell can be clearly mapped by EELS; however Ga and As signals are noisy. In order to assure the core composition, we utilized an individual nanotrough where the shell was locally discontinuous ( $c f$. Fig. 1(i)). In this case, one can appreciate on the EELS maps the increasing Ga and As signals on the uncovered area. The EELS profile shown in Fig. 1(j) has been obtained along the red arrow direction.

\subsection{Electrical measurements}

For the following magnetotransport studies we transferred nanotubes to a silicon wafer covered with $500 \mathrm{~nm}$ thick silicon oxide for electrical isolation. Single nanotubes were contacted with four $\mathrm{Cr} / \mathrm{Au}$ probes using an electron beam lithography based process (Fig. 1(c)). To obtain an interface resistance as low as a few Ohms we cleaned the nickel surface through in situ ion milling before evaporation of the adhesion layer ( $5 \mathrm{~nm}$ thick $\mathrm{Cr}$ ) and metal film (300 nm thick $\mathrm{Au}$ ) and lift-off processing. We performed four-point probe measurements of the nanotube resistance by applying the current $I$ at contacts 1 and 4 and measuring the voltage $V$ at contacts 2 and 3 (Fig. 1(d)). In order to improve the signal-to-noise ratio, we used either a nanovoltmeter and a current source operating in current-reversal mode or a lock-in amplifier to modulate $I$ and detect phasesensitively the voltage $V$. The current amplitude amounted to 4 $\mu \mathrm{A}$. Assuming a shell thickness of $40 \mathrm{~nm}$, this value corresponded to a current density of about $10^{4} \mathrm{~A} \mathrm{~cm}^{-2}$.

All data presented in the following are taken from one individual tube. Data taken from a second nanotube are presented at the end of Section 4.2. The electrical properties were studied by resistance measurements from room temperature down to $1.6 \mathrm{~K}$. At room temperature the specific resistivity $\rho$ was about $(25 \pm 10)$ $\mu \Omega \mathrm{cm}$. At $1.6 \mathrm{~K}$ we obtained $\rho=(5 \pm 2) \mu \Omega \mathrm{cm}$. This value is smaller than of the planar Ni nanowires reported by Hong and Giordano $^{33}$ and substantiates the good quality of the ALDgrown Ni shell.

\subsection{Magnetotransport measurements}

The magnetic properties were investigated by magnetotransport studies of field orientations parallel and perpendicular to the tube axis. The sample was cooled down to a temperature $T=1.6 \mathrm{~K}$ in a cryostat with a superconducting magnet providing an axial field $\mu_{0} \boldsymbol{H}$ of up to 9 Tesla. The sample holder allows us to vary the orientation of the nanotube with respect to $\boldsymbol{H}$ at low temperatures. In the course of a sweep, the resistance occasionally increased by an individual jump of $10-15 \mathrm{~m} \Omega$, which neither depended on the magnetic field nor was hysteretic. Such jumps were attributed to resistance changes in nanotroughs through electromigration. The resistance curves were corrected for such occasional effects. The large magnetic field $\boldsymbol{H}$ allowed us to saturate the nanotube magnetization $\boldsymbol{M}$ under different field orientations. This is a prerequisite to quantify the AMR effect. At the same time the low temperature enables us to be close to the condition $T=0$ used for the theoretical predictions.
We start by presenting the magnetoresistance and AMR effect for $\boldsymbol{H}$ applied parallel to the long nanotube axis. Before applying a magnetic field for the first time, the resistance was measured to be $17.936 \mathrm{~m} \Omega$. Then the magnetic state was saturated in the longitudinal direction by a magnetic field of $\mu_{0} \boldsymbol{H}=-1 \mathrm{~T}$. Fig. 2 shows the typical field-dependent behavior: $R(\boldsymbol{H})=V(\boldsymbol{H}) / I$ vs. $\boldsymbol{H}$ for $\mu_{0}|\boldsymbol{H}| \leq 1 \mathrm{~T}$. Towards larger fields, the nanotube exhibits a positive magnetoresistance. The resistance does not saturate up to $9 \mathrm{~T}$. The increase of the resistance for $\mu_{0} \boldsymbol{H}>1 \mathrm{~T}$ is attributed to the well-known Lorentz magnetoresistance. ${ }^{43}$ To analyze the AMR effect we thus focus on data taken for $\mu_{0}|\boldsymbol{H}| \leq 1 \mathrm{~T}$. At $1 \mathrm{~T}$, the resistance is $R_{\|, \max }=17.985 \Omega$. When decreasing $\mu_{0} \boldsymbol{H}$ from 1 T, $R$ deviates from $R_{\|, \text {max }}$ over a broad field regime ranging from about -0.2 to $+0.2 \mathrm{~T}$. This means that microscopic magnetic moments tilt away from the longitudinal direction, thereby reducing the spin-dependent scattering following the expression: ${ }^{44}$

$$
\rho(\theta)=\rho_{\perp}+\left(\rho_{\|}-\rho_{\perp}\right) \cos ^{2}(\theta) .
$$

Here, $\theta$ is the angle between the direction of current $I$ and magnetization $\boldsymbol{M}$. At small fields, the magnetoresistance is found to be hysteretic. This means that the magnetization at $\boldsymbol{H}=$ 0 depends on the magnetic history. ${ }^{33-35} \mathrm{We}$ attribute the minima $R_{\|, \text {min }}$ in $R_{\|}(\boldsymbol{H})$ to the coercive field amounting to $\mu_{0}\left|\boldsymbol{H}_{\mathrm{c}}\right| \approx$ $17 \mathrm{mT}$. The maximum resistance change is found to be $\delta_{\max } R_{\|}=$ $R_{\|, \max }-R_{\|, \min }=(48 \pm 2) \mathrm{m} \Omega$ as shown in Fig. 2 . In minor loop measurements (not shown) the resistance is found to remain constant and non-hysteretic, if we stay with $\boldsymbol{H}$ in the regime $\mu_{0}|\boldsymbol{H}| \lesssim 15 \mathrm{mT}$. Increasing the reversal field beyond $15 \mathrm{mT}$ we regain hysteretic behavior in $R(\boldsymbol{H})$. The hysteretic behavior and the minor loop measurements suggest an incoherent reversal mode of the nanotube.

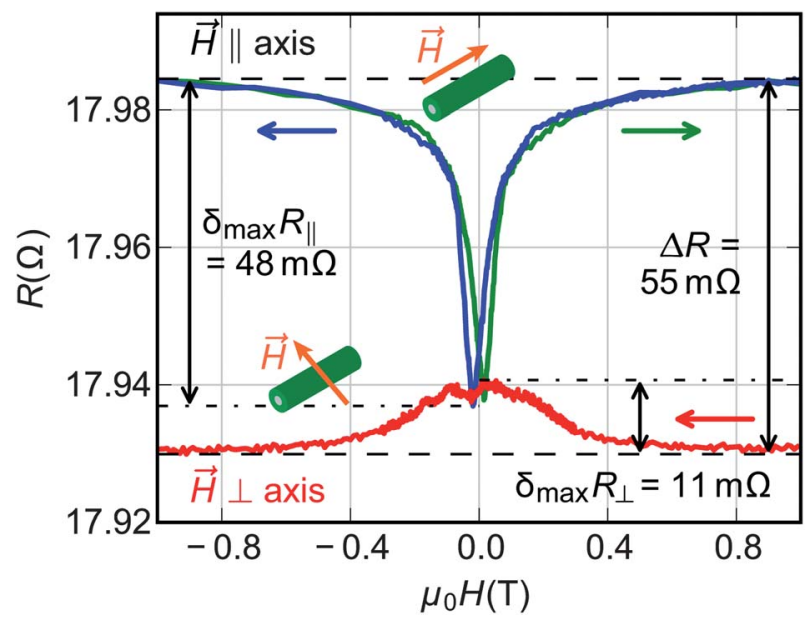

Fig. 2 Resistance of a $10 \mu \mathrm{m}$ long $\mathrm{Ni}$ nanotube segment for magnetic field sweeps in positive (green) and negative (blue) directions when $\boldsymbol{H}$ is parallel to the long axis (upper curves). The magnetoresistance is hysteretic and positive at large fields. We extract a coercive field of $\mu_{0} \boldsymbol{H}_{\mathrm{c}}$ $\approx 17 \mathrm{mT}$ from the data. The absolute variation is $\delta_{\max } R_{\|}=48 \mathrm{~m} \Omega$. For $\boldsymbol{H}$ perpendicular to the long nanotube axis the magnetoresistance is negative to large fields with $\delta_{\max } R_{\perp}=11 \mathrm{~m} \Omega$. Overall the relative AMR effect amounts to $\Delta R / R_{\perp, \min }=0.3 \%$ in the given Ni nanotube. 
Before we analyze the data in the parallel field configuration in more detail it is instructive to discuss the magnetoresistive behavior in perpendicular field. For this, we turn the direction of the sample in the cryostat at low temperatures and zero field after saturation at $-1 \mathrm{~T}$. The typical magnetoresistive behavior is shown in Fig. 2 (bottom curve). Starting from $\boldsymbol{H}=0, R(\boldsymbol{H})$ is found to decrease by $\delta_{\max } R_{\perp}=(11 \pm 2) \mathrm{m} \Omega$ up to $\mu_{0} \boldsymbol{H}=1 \mathrm{~T}$. The magnetoresistance is thus negative. It exhibits a relatively steep slope $R$ vs. $\boldsymbol{H}$ for $\mu_{0}|\boldsymbol{H}| \leq 0.4 \mathrm{~T}$. We attribute the field value of $0.4 \mathrm{~T}$ to the shape anisotropy field $\boldsymbol{H}_{\text {ani. For }}|\boldsymbol{H}|>\boldsymbol{H}_{\text {ani, }}$, the magnetization $\boldsymbol{M}$ of the device becomes aligned with $\boldsymbol{H}$ so that $\boldsymbol{M}$ is perpendicular to the applied current $I$. As a consequence, $R_{\perp}$ takes a minimum of $R_{\perp, \min }=17.930 \Omega$ at $1 \mathrm{~T}$. The overall resistance change $\delta_{\max } R_{\perp}=(11 \pm 2) \mathrm{m} \Omega$ is significantly smaller than $\delta_{\max } R_{\|}=(48 \pm 2) \mathrm{m} \Omega$ observed for parallel fields. Considering the resistance data from Fig. 2 we calculate the maximum relative AMR effect to be

$$
\Delta R / R_{\perp, \min }=\frac{R_{\|, \max }-R_{\perp, \min }}{R_{\perp, \min }}=0.3 \% .
$$

This is a reasonable value compared to the AMR effect observed on the ALD-grown planar Ni films. It is smaller by a factor of about 2. We attribute this discrepancy to the nanotroughs observed in Fig. 1. They locally reduce the cross-section of the nanotube. In contrast to the thin film, the current might not be able to percolate around the nanotroughs. The series of nanotroughs increases the specific resistivity which enters the denominator of eqn (3) via $R_{\perp \text {, min }}$ and thereby reduces the overall MR value.

Interestingly, $R$ is hysteretic in perpendicular fields as well. Fig. 3 shows field-dependent data (symbols) taken in minor loops between -0.17 and $+0.17 \mathrm{~T}$ where $\boldsymbol{H}$ was varied in small increments of $1 \mathrm{mT}$. For both sweep directions, field regions are found where $R$ takes a local minimum. Coming from, e.g., $+0.17 \mathrm{~T}$ and

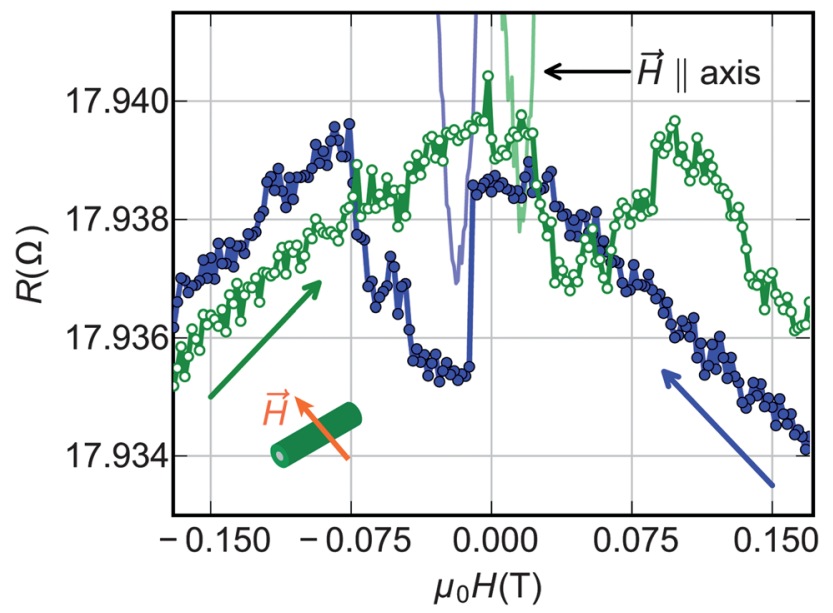

Fig. 3 Magnetoresistance data (symbols) for field sweeps between -0.17 and $+0.17 \mathrm{~T}$ when $\boldsymbol{H}$ is perpendicular to the nanotube: blue filled (green open) symbols show a sweep of $\boldsymbol{H}$ in the negative (positive) direction. For clarity symbols are interconnected by lines. The nanotube exhibits an overall decrease of $R$ for increasing fields and characteristic local minima at small opposing fields in the range of $0.010 \mathrm{~T} \lesssim\left|\mu_{0} \boldsymbol{H}\right| \lesssim 0.075 \mathrm{~T}$. For comparison, the lines show the major loop data presented in Fig. 2 for the $\boldsymbol{H} \|$ axis. Large arrows indicate sweep directions of $\boldsymbol{H}$. going to negative fields the resistance is found to drop abruptly by almost $3 \mathrm{~m} \Omega$ at $-0.010 \mathrm{~T}$. It remains small until $-0.075 \mathrm{~T}$ where $R$ regains a large value within the range of a few $\mathrm{mT}$. Note that the absolute value of the local minimum is larger than $R_{\perp, \min }$ at $\mu_{0} \boldsymbol{H}=1 \mathrm{~T}$. For further decreasing field, $R$ follows the negative magnetoresistance already seen in Fig. 2. The drop and local minimum in $R$ for small opposing fields are reproducible features for successive field sweeps. Depending on the exact reversal field the relevant field region is found to vary slightly.

\section{Discussion}

\subsection{AMR effect in nanotubes: development of a classification scheme}

We start by introducing the well-known characteristics of the AMR effect in planar thin films and discuss what should be expected in a nanotube configuration. For the AMR effect, the angle $\theta$ between the direction of current $I$ and magnetization $\boldsymbol{M}$ is decisive. The specific resistivity $\rho$ varies due to spin-dependent scattering provoked by spin-orbit coupling. In a ferromagnetic bulk material the field-dependent resistivity follows eqn (2). Because $\rho_{\|}>\rho_{\perp}$ in Ni, $\rho$ is expected to be at maximum when $I$ and $\boldsymbol{M}$ are collinear. It is at a minimum when the magnetization is perpendicular to the current. Rijks et al. ${ }^{35}$ showed that the relative magnetoresistance (MR) effect is modified by boundary scattering in a planar thin film. As a function of $\theta$ they find a different behavior for $\boldsymbol{M}$ staying in the film plane or pointing perpendicular to the film boundary. The qualitative behavior $R(\theta)$ is sketched in Fig. 4(a) as extracted from ref. 35. In Fig. 4(b) we illustrate the magnetic states of the thin film (left) at

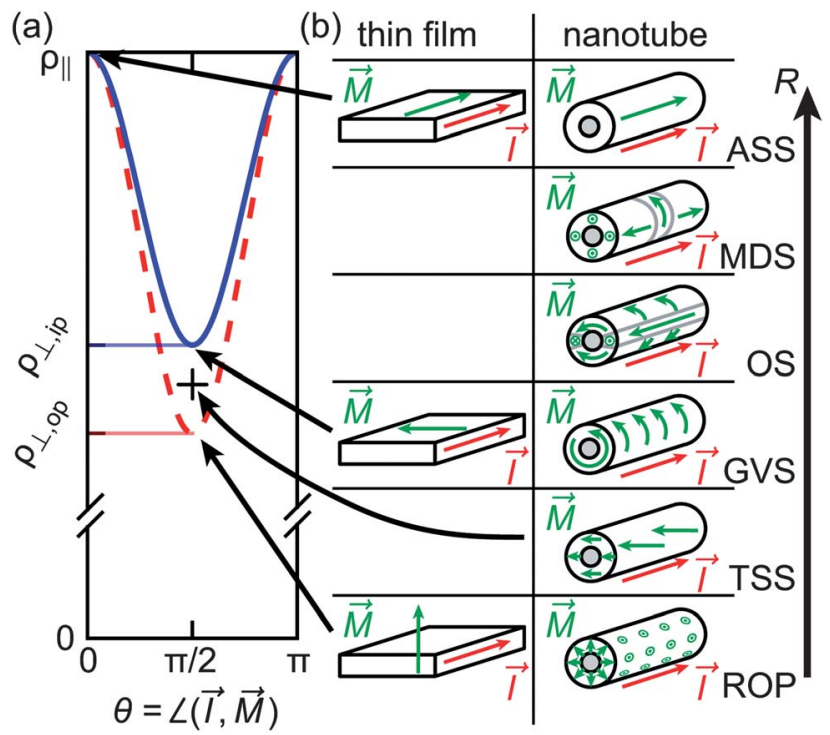

Fig. 4 (a) Theoretical variation of the specific resistivity $\rho$ of a thin film as a function of the angle between $I$ and $\boldsymbol{M}$ for rotation of $\boldsymbol{M}$ in plane (blue, solid) and out-of-plane (red, dashed) [after Rijks et al. ${ }^{35}$ ]. (b) Relevant magnetic states in the thin film (left) compared to configurations in a nanotube (right) as discussed in the text. Configurations are ordered such that the resistance increases from bottom to top. Arrows indicate the orientations of magnetic moments and current. The semiconductor core is shown in gray. 
characteristic points of the $R(\theta)$ dependencies. We will show that they allow us to classify the magnetic states of the nanotube (right). For the thin film, the largest resistance occurs when the magnetization is collinear with the current, resulting in $\rho=$ $\max (\rho)=\rho_{\|}$. Now, if we compare the resistivity in the case where $\boldsymbol{M}$ is in-plane $\left(\rho=\rho_{\perp, \text { ip }}\right)$ or out-of-plane $\left(\rho=\rho_{\perp, \mathrm{op}}\right)$, the smallest resistance is achieved for an out-of-plane magnetization, due to the modified boundary scattering. Such a discrepancy does not occur in eqn (2) for bulk materials in which boundary scattering is not relevant. According to Rijks et al., ${ }^{35}$ a measurable difference between in-plane and out-of-plane AMR ratios is present for film thicknesses below a critical value $t_{\mathrm{c}}$ of approximately $100 \mathrm{~nm}$.

We now turn to the discussion of the nanotube, for which we consider the current to be always parallel to the long nanotube axis. The shell thickness is about $40 \mathrm{~nm}$ and smaller than $t_{\mathrm{c}}$. We thus attribute the MR behavior of the nanotube to the effect which was elaborated by Rijks et al. As in the thin film case, the maximum resistance is achieved when the magnetization is parallel to the current. We call this the axially saturated state (ASS). Fig. 4 would suggest the same resistivity for the nanotube and the thin film. Interestingly, the minimum resistivity $\rho_{\perp, \mathrm{op}}$ obtained for the thin film would correspond to a state of the nanotube in which the magnetic moments obey a radially aligned out-of-plane configuration (ROP). Such a state (bottom-most graph in Fig. 4(b)) might be created by a tailored magnetocrystalline or interfacial anisotropy. For a nanotube prepared from an isotropic ferromagnet as considered here, the minimum resistivity state of Fig. 4(a) cannot be achieved. For such a nanotube, the lowest resistivity is obtained in a transversally saturated state (TSS), in which all magnetic moments are aligned along a direction perpendicular to the long nanotube axis. Such a configuration is attained at large magnetic fields. Here, it is interesting to note that due to the curved surface, only a small portion of the moments point perpendicularly to the nanotube surface. The relevant resistivity falls between $\rho_{\perp \text {,ip }}$ and $\rho_{\perp, \text { op }}$ and is marked with a cross in Fig. 4(a). We now consider the intermediate magnetization states between ASS and TSS. Slightly above the resistivity of the TSS, we find the global vortex state (GVS), in which the moments follow the circumference of the nanotube and are aligned in the azimuthal direction. Such a state has been predicted to occur in equilibrium for nanotubes above a certain diameter. ${ }^{21}$ The resistivity of the global vortex state coincides with the resistivity $\rho_{\perp \text {,ip }}$ of the thin film.

In close analogy to the onion-state (OS) in ferromagnetic ring structures, ${ }^{11-13}$ we propose the existence of a comparable state in magnetic nanotubes. Here, the two halves of the nanotube exhibit parallel magnetic moments being aligned in the azimuthal direction. The two halves are separated by domain walls. To minimize the stray field energy the moments in the domain walls are expected to align with the long axis and in opposite direction for both domains. With this, the overall resistivity will increase according to eqn (2). The resistivity of OS is thus expected to be larger compared to GVS (Fig. 4(b)). For the vortex state in an individual permalloy ring, a higher resistance compared to that of the onion state was observed. ${ }^{13}$ This was due to the orientation of the current which was in the plane of the magnetic moments. In contrast, the current is perpendicular to the moments in our nanotube, leading to the opposite behavior in $R(\boldsymbol{H})$.
At an even higher resistivity but still below the ASS, we classify the multi-domain state (MDS) formed by a series of domains in the ASS configuration of opposite directions separated by domain walls. Domain walls might be in a TSS- or GVS-like configuration. ${ }^{22}$

\subsection{Magnetic states assigned to measured resistance values}

We now turn to the discussion of magnetic states observed with the magnetic field applied in a direction parallel or perpendicular to the long nanotube axis. We use the classification developed in Fig. 4 to attribute magnetic states to the measured resistance values as sketched in Fig. 5. Experimentally, we obtain the lowest resistance in the magnetic nanotube when we generate the transversely saturated state (TSS) by applying $\mu_{0} \boldsymbol{H}=1 \mathrm{~T}>$ $\mu_{0} \boldsymbol{H}_{\text {ani }}$ in a direction perpendicular to the long axis (Fig. 2). When reducing the magnetic field, the TSS is found to be unstable. The demagnetization field is largest where the surface normal is parallel to the external magnetic field. At these points, the magnetic moments tilt away from the field direction if $\boldsymbol{H}<$ $\boldsymbol{H}_{\text {ani }}$ and form head-to-head or tail-to-tail domain walls. To minimize the stray-field energy, moments will most likely turn into an axial direction, provoke a domain wall and form the OS

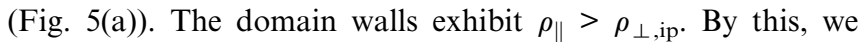
explain the gradual increase in $R$ when reducing $\boldsymbol{H}$ in the perpendicular field direction. We propose the OS as the stable configuration at $\boldsymbol{H}=0$. The increased value of $R$ at $\boldsymbol{H}=$ 0 depends on the total width of segments with $\rho_{\|}>\rho_{\perp, \text { ip. }}$. The overall specific resistivity is certainly larger than $\rho=\rho_{\perp \text {,ip. In an }}$ opposing magnetic field, large parts of the nanotube are expected to switch irreversibly and form a GVS. In the vortex state, the small specific resistivity $\rho_{\perp, \text { ip }}$ is realized around the nanotube. Following this argument, we attribute the abrupt jump of reduced $R$ in Fig. 3 to the creation of the GVS ( $c f$. Fig. 5(a)). This state is stable in a small field region and has a resistance which is
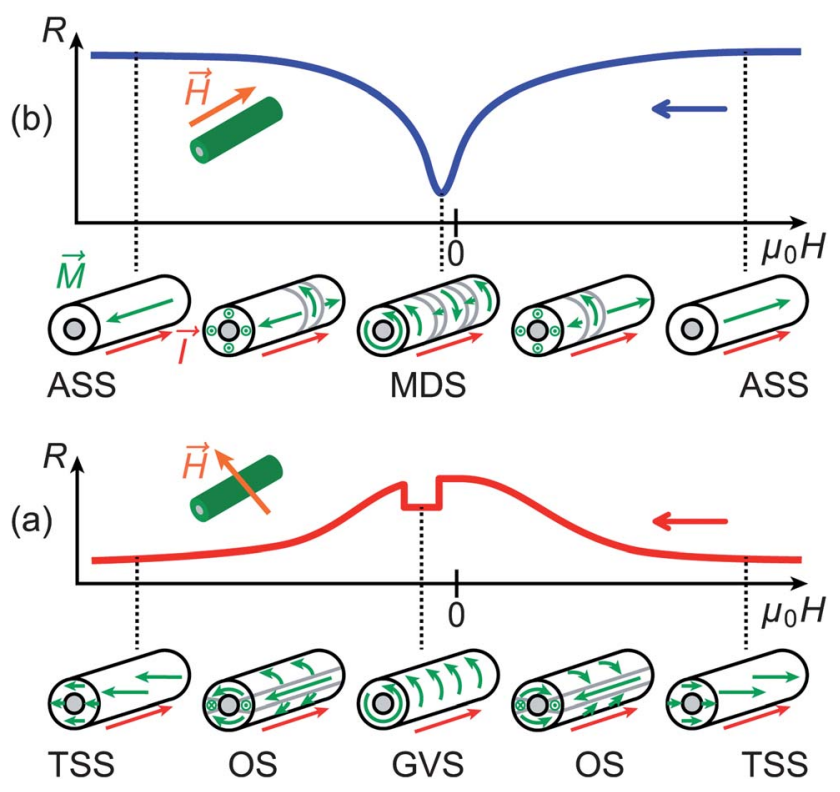

Fig. 5 Magnetic states schematically attributed to characteristic resistance values in (a) a perpendicular and (b) a parallel magnetic field. 
between those of TSS and OS at $\boldsymbol{H}=0$. At a field of $\mu_{0}|\boldsymbol{H}| \approx$ $0.075 \mathrm{~T}$, the energy attributed to the misalignment of the spins in the external field will be higher than the energy gain due to the flux-closure configuration. At this field, the configuration changes abruptly back to the OS with nanotube halves being aligned in the negative field direction.

It is now interesting to address the reversal in a field $\boldsymbol{H}$ applied parallel to the long axis. In Fig. 2 and 3 we observe that $R\left(\boldsymbol{H}_{\mathrm{c}}\right)$ is almost as small as the resistance of the vortex-like state discussed above. Comparing $\delta_{\max } R_{\|}=48 \mathrm{~m} \Omega$ from Fig. 2 with the maximum absolute change $\Delta R=59 \mathrm{~m} \Omega$, we calculate the relative amount of magnetic moments being perpendicular to $I$ during reversal to be $87 \%$ of the total magnetization. In ref. 22 the reversal mechanism was investigated theoretically assuming an ideal nanotube without surface roughness. For the geometrical parameters realized by our nanotubes, the authors predicted an abrupt reversal to occur via a single vortex wall. In the real nanotube, we find the resistance to change gradually with $\boldsymbol{H}$ in a wide field region. At this point we cannot decide whether the reversal occurs via a global vortex state or segments of vortexlike domain walls (vortex walls) separated by ASS domains. The scenario of vortex walls sketched in Fig. 5(b) seems to be more likely considering the surface roughness of our nanotubes. Vortex walls might enter the nanotube in a sequential manner and thereby explain the wide field region where $R$ deviates from $R_{\|, \text {max }}$. The gradual change of $R$ implies the creation, movement and pinning of a large number of vortex walls where locally $\boldsymbol{M}$ is perpendicular to $I$. The minimum resistance $R_{\|, \text {min }}$ is achieved at $\boldsymbol{H}=\boldsymbol{H}_{\mathrm{c}}$ when the maximum number of domain walls reside between the voltage probes.

In the scenarios discussed above, the absolute resistance changes $\Delta R$ between ASS and TSS as well as $\delta_{\max } R_{\perp}$ between VS and TSS should depend little on the exact number of nanotroughs. In contrast, we expect $\delta_{\max } R_{\|}$to depend on the number of nanotroughs serving as pinning sites. Magnetotransport experiments performed on a further nanotube with similar geometrical parameters provided the following data at $4 \mathrm{~K}: \Delta R=$ $51 \mathrm{~m} \Omega, \delta_{\max } R_{\perp}=12 \mathrm{~m} \Omega$, and $\delta_{\max } R_{\|}=22 \mathrm{~m} \Omega$. The number of nanotroughs and their microscopic shape were different compared to those of the device in Fig. 2. However, only the value $\delta_{\max } R_{\|}$deviated substantially (by a factor of 2 ) from values obtained on the nanotube presented above. This is consistent with the argument that we expect nanotroughs to change mainly $\delta_{\max } R_{\|}$.

\section{Conclusion}

We have discussed magnetotransport experiments performed on individual ferromagnetic nanotubes. For parallel field orientation, the vortex wall reversal mode predicted by theoretical studies seems to be consistent with the anisotropic magnetoresistance data. The reversal occurs in segments in a sequential manner. For a magnetic field applied perpendicular to the long axis we suggest onion and vortex states to form during the reversal. This field geometry has not been considered theoretically before. In this work, we have developed a classification scheme for magnetic states of nanotubes which is derived from the AMR effect known for planar films. This scheme allows one to relate resistance changes to different magnetic states. For memory applications, it would be extremely important to control all the different magnetization configurations depending on the magnitude and orientation of the magnetic field applied. Interestingly, both, the ROP and GVS, lead to zero magnetization but significantly different stray fields. In the GVS the stray field is zero, avoiding magnetostatic interaction between memory elements.

\section{Acknowledgements}

The authors kindly thank Kornelius Nielsch, Julien Bachmann and Tobias Stückler for discussions and experimental support. The research leading to these results has received funding from the European Community's Seventh Framework Programme (FP7/2007-2013) under grant agreement no. 228673 MAGNONICS. DR, MH, ER and AFM acknowledge financial support from the NCCR QSIT, ERC 'Upcon' and the School of Basic Sciences at Ecole Polytechnique Federale de Lausanne. This work was supported by the Spanish MICINN Projects MAT2010-15138 and CSD2009-00013. JA also acknowledges Generalitat de Catalunya 2009-SGR-770 and XaRMAE.

\section{References}

1 S. E. Thompson and S. Parthasarathy, Mater. Today, 2006, 9, 20-25, DOI: 16/s1369-7021(06)71539-5.

2 S. S. P. Parkin, M. Hayashi and L. Thomas, Science, 2008, 320, 190 194, DOI: $10.1126 /$ science.1145799.

3 S. Neusser and D. Grundler, Adv. Mater., 2009, 21, 2927-2932, DOI: 10.1002/adma.200900809.

4 V. V. Kruglyak, S. O. Demokritov and D. Grundler, J. Phys. D: Appl. Phys., 2010, 43, 264001, DOI: 10.1088/0022-3727/43/26/264001.

5 International Technology Roadmap for Semiconductors (ITRS), 2009, http://www.itrs.net/Links/2009ITRS/Home2009.htm.

6 A. Khitun, M. Bao and K. L. Wang, J. Phys. D: Appl. Phys., 2010, 43, 264005, DOI: $10.1088 / 0022-3727 / 43 / 26 / 264005$.

7 Q. A. Pankhurst, J. Connolly, S. K. Jones and J. Dobson, J. Phys. D: Appl. Phys., 2003, 36, R167-R181, DOI: 10.1088/0022-3727/36/13/ 201

8 S. J. Son, J. Reichel, B. He, M. Schuchman and S. B. Lee, J. Am. Chem. Soc., 2005, 127, 7316-7317, DOI: 10.1021/ja0517365.

9 E. Amstad, S. Zurcher, A. Mashaghi, J. Y. Wong, M. Textor and E. Reimhult, Small, 2009, 5, 1334-1342, DOI: 10.1002/ smll.200801328.

10 D.-H. Kim, E. A. Rozhkova, I. V. Ulasov, S. D. Bader, T. Rajh, M. S. Lesniak and V. Novosad, Nat. Mater., 2010, 9, 165-171, DOI: $10.1038 /$ nmat 2591 .

11 J. Rothman, M. Kläui, L. Lopez-Diaz, C. A. F. Vaz, A. Bleloch, J. A. C. Bland, Z. Cui and R. Speaks, Phys. Rev. Lett., 2001, 86, 1098-1101, DOI: 10.1103/physrevlett.86.1098.

12 F. J. Castaño, C. A. Ross, C. Frandsen, A. Eilez, D. Gil, H. I. Smith, M. Redjdal and F. B. Humphrey, Phys. Rev. B: Condens. Matter, 2003, 67, 184425, DOI: 10.1103/PhysRevB.67.184425.

13 J. Podbielski, F. Giesen, M. Berginski, N. Hoyer and D. Grundler, Superlattices Microstruct., 2005, 37, 341-348, DOI: 10.1016/ j.spmi.2004.12.006.

14 Z. K. Wang, M. H. Kuok, S. C. Ng, D. J. Lockwood, M. G. Cottam, K. Nielsch, R. B. Wehrspohn and U. Gösele, Phys. Rev. Lett., 2002, 89, 027201, DOI: 10.1103/PhysRevLett.89.027201.

15 J. Topp, J. Podbielski, D. Heitmann and D. Grundler, Phys. Rev. B: Condens. Matter Mater. Phys., 2008, 78, 024431, DOI: 10.1103/ PhysRevB.78.024431

16 E. Vogel, P. Vargas, D. Altbir and J. Escrig, Nanotubes and Nanowires, CRC Press, 2010.

17 R. Hertel and J. Kirschner, J. Magn. Magn. Mater., 2004, 278, L291L297, DOI: 10.1016/j.jmmm.2004.02.032.

18 J. Escrig, M. Daub, P. Landeros, K. Nielsch and D. Altbir, Nanotechnology, 2007, 18, 445706, DOI: 10.1088/0957-4484/18/44/ 445706. 
19 J. Escrig, P. Landeros, D. Altbir, E. Vogel and P. Vargas, J. Magn. Magn. Mater., 2007, 308, 233-237, DOI: 16/j.jmmm.2006.05.019.

20 P. Landeros, P. R. Guzmán, R. Soto-Garrido and J. Escrig, J. Phys. D: Appl. Phys., 2009, 42, 225002, DOI: 10.1088/0022-3727/42/22/ 225002.

21 P. Landeros, O. J. Suarez, A. Cuchillo and P. Vargas, Phys. Rev. B. Condens. Matter Mater. Phys., 2009, 79, 024404, DOI: 10.1103/ PhysRevB.79.024404

22 P. Landeros, S. Allende, J. Escrig, E. Salcedo, D. Altbir and E. E. Vogel, Appl. Phys. Lett., 2007, 90, 102501, DOI: 10.1063/ 1.2437655 .

23 P. Landeros and A. S. Núñez, J. Appl. Phys., 2010, 108, 033917, DOI: 10.1063/1.3466747.

24 J. Bachmann, J. Jing, M. Knez, S. Barth, H. Shen, S. Mathur, U. Gösele and K. Nielsch, J. Am. Chem. Soc., 2007, 129, 95549555, DOI: $10.1021 / \mathrm{ja0} 072465 \mathrm{w}$.

25 M. Daub, M. Knez, U. Goesele and K. Nielsch, J. Appl. Phys., 2007, 101, 09J111, DOI: $10.1063 / 1.2712057$

26 J. Bachmann, J. Escrig, K. Pitzschel, J. M. M. Moreno, J. Jing, D. Görlitz, D. Altbir and K. Nielsch, J. Appl. Phys., 2009, 105, 07B521, DOI: 10.1063/1.3074109.

27 A. Rudolph, M. Soda, M. Kiessling, T. Wojtowicz, D. Schuh, W. Wegscheider, J. Zweck, C. Back and E. Reiger, Nano Lett., 2009, 9, 3860-3866, DOI: 10.1021/n19020717.

28 Y. T. Chong, D. Görlitz, S. Martens, M. Y. E. Yau, S. Allende, J. Bachmann and K. Nielsch, Adv. Mater., 2010, 22, 2435-2439, DOI: 10.1002/adma.200904321.

29 O. Albrecht, R. Zierold, S. Allende, J. Escrig, C. Patzig, B. Rauschenbach, K. Nielsch and D. Görlitz, J. Appl. Phys., 2011, 109, 093910, DOI: $10.1063 / 1.3583666$.

30 J. Escrig, J. Bachmann, J. Jing, M. Daub, D. Altbir and K. Nielsch, Phys. Rev. B: Condens. Matter Mater. Phys., 2008, 77, 214421, DOI: 10.1103/PhysRevB.77.214421.

31 S. Barth, S. Estrade, F. Hernandez-Ramirez, F. Peiro, J. Arbiol, A. Romano-Rodriguez, J. R. Morante and S. Mathur, Cryst. Growth Des., 2009, 9, 1077-1081, DOI: 10.1021/cg8009095.

32 C. Butschkow, E. Reiger, S. Geißler, A. Rudolph, M. Soda, D. Schuh, G. Woltersdorf, W. Wegscheider and D. Weiss, Arxiv preprint arXiv:1110.5507, 2011.
33 K. Hong and N. Giordano, Phys. Rev. B: Condens. Matter, 1995, 51, 9855-9862, DOI: 10.1103/PhysRevB.51.9855.

34 J.-E. Wegrowe, D. Kelly, A. Franck, S. E. Gilbert and J.-P. Ansermet, Phys. Rev. Lett., 1999, 82, 3681-3684, DOI: 10.1103/ PhysRevLett.82.3681.

35 T. G. S. M. Rijks, R. Coehoorn, M. J. M. de Jong and W. J. M. de Jonge, Phys. Rev. B: Condens. Matter, 1995, 51, 283-291, DOI: 10.1103/PhysRevB.51.283.

36 E. Uccelli, J. Arbiol, C. Magen, P. Krogstrup, E. Russo-Averchi, M. Heiss, G. Mugny, F. Morier-Genoud, J. Nygard, J. R. Morante and A. Fontcuberta i Morral, Nano Lett., 2011, 11, 3827-3832, DOI: $10.1021 / \mathrm{nl} 201902 \mathrm{w}$.

37 E. Russo-Averchi, M. Heiss, L. Michelet, P. Krogstrup, J. Nygard, C. Magen, J. R. Morante, E. Uccelli, J. Arbiol and A. Fontcuberta i Morral, Nanoscale, 2012, 4, 1486-1490, DOI: 10.1039/c2nr11799a.

38 M. Knez, K. Nielsch and L. Niinistö, Adv. Mater., 2007, 19, 34253438, DOI: 10.1002/adma.200700079.

39 R. Huber, T. Schwarze, P. Berberich, T. Rapp and D. Grundler, Atomic layer deposition for the fabrication of magnonic metamaterials, in Metamaterials 2011: The Fifth International Congress on Advanced Electromagnetic Materials in Microwaves and Optics, Metamorphose-VI, ISBN 978-952-67611-0-7, p. 588, 2011.

40 T. Stückler, Magnetowiderstand von ferromagnetischen Filmen auf $3 D$ beschichteten Substraten, Bachelor Thesis, Physik Department, Technische Universität, München,2011.

41 D. Spirkoska, J. Arbiol, A. Gustafsson, S. Conesa-Boj, I. Zardo, M. Heigoldt, M. H. Gass, A. L. Bleloch, S. Estrade, F. Peiro, J. Morante, G. Abstreiter, L. Samuelson and A. F. I. Morral, Phys. Rev. B: Condens. Matter Mater. Phys., 2009, 80, 245325, DOI: 10.1103/PhysRevB.80.245325.

42 A. Fontcuberta i Morral, D. Spirkoska, J. Arbiol, M. Heigoldt, J. R. Morante and G. Abstreiter, Small, 2008, 4, 899-903, DOI: 10.1002/smll.200701091.

43 F. C. Schwerer and J. Silcox, Phys. Rev. Lett., 1968, 20, 101-103, DOI: 10.1103/PhysRevLett.20.101.

44 T. McGuire and R. Potter, IEEE Trans. Magn., 1975, 11, 1018-1038, DOI: 10.1109/TMAG.1975.1058782. 